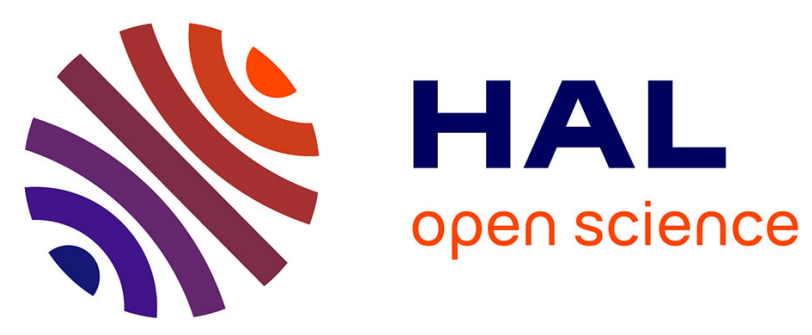

\title{
Une étude exploratoire des représentations de la gratuité et de ses effets sur le comportement des publics des musées et des monuments.
}

Marine Le Gall-Ely, Caroline Urbain, Anne Gombault, Dominique Bourgeon, Christine Petr

\section{To cite this version:}

Marine Le Gall-Ely, Caroline Urbain, Anne Gombault, Dominique Bourgeon, Christine Petr. Une étude exploratoire des représentations de la gratuité et de ses effets sur le comportement des publics des musées et des monuments.. Recherche et Applications en Marketing (French Edition), 2007, 22 (2), pp.23-38. hal-00522834

\section{HAL Id: hal-00522834 \\ https://hal.science/hal-00522834}

Submitted on 1 Oct 2010

HAL is a multi-disciplinary open access archive for the deposit and dissemination of scientific research documents, whether they are published or not. The documents may come from teaching and research institutions in France or abroad, or from public or private research centers.
L'archive ouverte pluridisciplinaire $\mathbf{H A L}$, est destinée au dépôt et à la diffusion de documents scientifiques de niveau recherche, publiés ou non, émanant des établissements d'enseignement et de recherche français ou étrangers, des laboratoires publics ou privés. 


\section{Une étude exploratoire des représentations de la gratuité et de ses effets sur le}

\section{comportement des publics des musées et des monuments.}

Final draft, paru dans Recherche et Applications Marketing, 2007, 22, 2, 23-38.

\section{Auteurs}

Marine LE GALL-ELY

Maître de Conférences

Université de Rennes 2

Place du recteur Henri Le Moal

35043 Rennes Cedex

CRESS-Lessor EA2614 et ICI EA2652

㥜 : 0299655380

Mél : marine.legall@uhb.fr

Caroline URBAIN

Maître de Conférences

CRGNA Université de Nantes

Mél : caroline.urbain@wanadoo.fr

Anne GOMBAULT

Professeur à Bordeaux Ecole de Management

Responsable de la Chaire Arts, Culture et Management en Europe (CEREBEM)

Mél : anne.gombault@bordeaux-bs.edu

Dominique BOURGEON-RENAULT

Professeur à l'Université de Bourgogne

CERMAB - LEG / UMR CNRS 5118

Chercheur associé à la Chaire Arts, Culture \& Management in Europe (CEREBEM)

Mél : dominique.bourgeon@u-bourgogne.fr

Christine PETR

Maître de Conférences

CREM UMR CNRS 6211

Institut de Gestion de Rennes, Université de Rennes 1

Mél : christine.petr@univ-rennes1.fr

Les auteurs tiennent à remercier les quatre lecteurs anonymes qui ont contribué à une amélioration sensible de cet article, ainsi que Christian Pinson, rédacteur en chef, pour ses encouragements et suggestions. Ils remercient également Monique Zollinger et Karine Gallopel pour leurs relectures de la version initiale de ce projet. 


\section{Une étude exploratoire des représentations de la gratuité et de ses effets sur le comportement des publics des musées et des monuments.}

Résumé : Que représente la gratuité des musées et des monuments pour les publics français, visiteurs ou non-visiteurs? Quelles en sont les conséquences sur leurs représentations, leurs projets d'usage et leurs comportements vis-à-vis des musées et des monuments ? Cette recherche a tenté de répondre à ce questionnement, à l'aide d'une méthodologie de multiangulation croisant différentes méthodes de production et d'analyse des données. Les résultats montrent que la gratuité déstabilise les représentations des musées et des monuments qu'ont les publics, qu'elle est secondaire dans la construction et la réalisation d'un projet de visite et enfin que l'expérience de la visite gratuite peut permettre, pour certains publics, un apprentissage de la visite ainsi que l'appropriation de la mesure de gratuité. Nous concluons sur la nécessité, avant toute chose, d'accroître l'implication des publics dans l'activité de visite et le besoin d'accompagner la mesure de gratuité.

Mots clés : gratuité, prix, musées et monuments, multiangulation

\section{An exploratory study of free admission to museums and monuments : audiences'}

\section{perceptions and effects on visiting behaviors.}

Abstract : What perceptions of free admission to museums and monuments do French visiting and non-visiting audiences have ? What are the consequences of such perceptions on individuals' perceptions, visit-planning and behavior patterns in museums and monuments? Research conducted between 2002 and 2003 tried to answer these questions by multiangulation applied to data production and analysis. Results show that free admission alter the perceptions of museums and monuments, it is secondary in planning and implementation of visit-planning, and finally it can spark a learning process from the visit that results in the appropriation of a free admission scheme. Conclusions are related to the need to create involvement toward the visit and communicate on free admission at first. 
$\underline{\text { Key words }}$ : Free admission, price, museums and monuments, multiangulation 


\section{INTRODUCTION}

Objet de débats anciens et passionnés entre partisans d'un libre accès à la culture et promoteurs d'une logique financière pragmatique, la gratuité revient aujourd'hui sur le devant de la scène dans les musées et les monuments. C'est notamment le cas au Louvre en 1996, dans tous les musées et monuments historiques nationaux en 2000, dans les musées parisiens en 2002 et dans de nombreux musées municipaux comme à Caen, Dijon et Bordeaux ou départementaux comme dans l'Isère. Même s'ils ont renoncé par réalisme au «tout-gratuit », les responsables des politiques culturelles restent fortement attachés à la gratuité en tant que symbole originel et valeur idéale du musée (Gombault, 2002). Au Louvre tout comme dans les musées nationaux, la fréquentation des dimanches gratuits est supérieure d'environ $60 \%$ à celle observée les dimanches payants. Cet effet semble cependant se tasser au fil du temps et la démocratisation tant espérée se fait attendre (Fourteau, 2001 ; Octobre et Rouet, 2002). Audelà de ces quelques éléments relatifs à la fréquentation, il existe peu de données pour connaître les représentations des publics concernant la gratuité des musées et des monuments et ses effets sur les comportements de visite. Quelles représentations de la gratuité ont-ils et comment celles-ci sont-elles reliées à leurs représentations, projets d'usage et comportements de fréquentation de ces lieux ? C'est à cette problématique que cette recherche exploratoire a cherché à répondre.

Bien que, dans la recherche en marketing, le prix soit abordé sous ses multiples facettes (Desmet et Zollinger, 1997 ; Monroe, 1990 ; Zollinger, 2004), la gratuité est un objet de recherche quasiment ignoré (Gorn, Tse et Weinberg, 1990). Ce constat nous a conduit à donner un objectif exploratoire à cette recherche et à mettre en œuvre une stratégie de multiangulation $^{1}$ de la production et de l'analyse des données (Weick, 1989 ; Lewis et

\footnotetext{
${ }^{1}$ La multiangulation est le plus souvent utilisée comme un outil de validation des recherches qualitatives. Cependant quand elle est utilisée de façon systématique, elle devient une véritable stratégie de recherche, utilisée alors pour explorer les différentes dynamiques d'un phénomène social complexe, et permettre ainsi d'en donner une vue globale. C'est dans cette configuration qu'elle a été choisie dans cette recherche.
} 
Grimes, 1999). Elle permet de comparer de multiples sources de données empiriques et théoriques pour produire de la connaissance et garantit ainsi une validité et une fiabilité élevées des résultats. Quatre modes de production des données ont été retenus : des entretiens individuels, mode central complété par des entretiens de groupe, des observations sur site et un questionnaire. Nous avons abordé la gratuité sous les différentes formes qu'elles prenaient dans les musées et des monuments : gratuité pour tous (dimanches gratuits) ou catégorielle (exonérations ciblées pour les moins de 18 ans ou les chômeurs), gratuité permanente (musées de la ville de Paris par exemple) ou événementielle (Journées du Patrimoine, Nuit des musées), gratuité totale (accès à tous les lieux et œuvres) ou partielle (limitée aux expositions permanentes ou aux jardins des monuments).

Cette recherche a abouti à trois métapropositions à valeur d'hypothèses ${ }^{2}$ qui contribuent à comprendre la manière dont les publics se représentent la gratuité et les effets que ces représentations peuvent avoir sur le comportement de visite. D'abord, la gratuité est appréhendée par les publics à travers leurs représentations des musées et des monuments et elle interroge ces représentations. Ensuite, elle est secondaire dans la construction et la réalisation d'un projet de visite. Enfin, il semble que l'expérience de la visite gratuite peut permettre à certains visiteurs un apprentissage de la visite des musées et des monuments ainsi que l'appropriation de la mesure de gratuité.

La suite de cet article présente la revue de la littérature effectuée ainsi que la méthodologie employée, suivies de l'analyse et de la discussion des résultats. Il se conclut par les implications managériales de cette recherche pour les acteurs des institutions culturelles, ses limites et les voies de recherche à poursuivre relatives à l'utilisation de la gratuité dans le secteur culturel, dans les services et plus généralement pour tout produit.

REVUE DE LA LITTERATURE

\footnotetext{
${ }^{2}$ Une méta-proposition est un agrégat de propositions à valeur d'hypothèses, visant à un niveau de connaissance théorique générale sur la question abordée.
} 
D’emblée, notons que le terme «gratuit» est polysémique (Godbout et Caillé, 1992), signifiant tour à tour sans valeur d'échange (sans prix), sans valeur d'usage (sans utilité), sans rationalité (une affirmation sans preuve), généreux, gracieux (un cadeau) et enfin, pour celui qui donne, libre, sans obligation et sans exigence de retour, sens le plus contesté, notamment par différents courants de recherche sur le don $^{3}$. Si des philosophes (Hénaff, 2002), des sociologues à travers la notion de don (Godbout, 2000 ; Godbout et Caillé, 2002), ou encore certains économistes traitant des services rendus gratuitement entre personnes (Jouvenel, 2002) se sont intéressés à la gratuité, ils ne répondent pas aux problématiques qu'elle soulève en sciences de gestion. Ils ne traitent notamment pas des représentations ${ }^{4}$ qu'ont les individus de cette mesure et très peu de ses effets ${ }^{5}$. Nous avons alors cherché à savoir si, dans la littérature sur le comportement des publics des musées et des monuments, et plus généralement, sur le comportement du consommateur, des travaux permettaient d'éclairer nos questions de recherche, à savoir quelles sont les représentations de la gratuité et quels sont ses effets sur le comportement des publics, visiteurs et non-visiteurs.

\section{La gratuité et le comportement des publics des musées et des monuments}

Dans le champ des musées et des monuments, l'essentiel des travaux existants est consacré aux politiques de la gratuité, c'est à dire à la gratuité telle que les institutions se la représentent et telle qu'elles la mettent en œuvre (pour une revue, voir Gombault, Petr et alii, 2006). Les quelques études portant sur les publics traitent principalement de l'impact quantitatif de la gratuité sur la fréquentation. Elles montrent qu'il est positif à court terme, une

\footnotetext{
3 Sagot-Duvauroux (1995) remarque que «le donateur adresse son don de façon souveraine et crée éventuellement une dette, une dépendance, une réciprocité de celui qui reçoit. La gratuité est une mise à disposition anonyme ou quelconque. » Un acte gratuit aurait un caractère désintéressé, sans fondement, tandis que le don engendrerait une relation fondée sur la dépendance de celui qui reçoit (Godbout et Caillé, 1992 ; Mauss, 1923-1924).

${ }^{4}$ La représentation est entendue ici comme « une forme de connaissance, socialement élaborée et partagée ayant une visée pratique et concourant à la construction d'une réalité commune à un ensemble social » (Jodelet, 1997). Elle est à la fois «le produit et le processus d'une activité mentale par laquelle un individu ou un groupe reconstitue le réel auquel il est confronté et lui attribue une signification spécifique » (Abric, 1987).

${ }^{5}$ Les discours sur la gratuité restent du domaine de l'assertion courante du type «ce qui est gratuit ne vaut rien », « ce qui n'a pas de prix doit être gratuit » ou « rien n'est jamais gratuit ».
} 
sorte d' «effet lune de miel » (Dickenson, 1993; Bagdali, 1998) et neutre à moyen et long terme. Ce résultat conforte les travaux économiques qui démontrent que le prix est une variable secondaire dans le comportement du consommateur culturel et notamment du visiteur de musée ou de monument (O’Hare, 1975; O’Hagan, 1995 ; Bailey et al. 1997). D'une manière générale, c'est le manque d'implication des publics qui constitue le principal frein à la visite et non le prix, qui intervient dans la décision de visite à partir du moment où le visiteur potentiel est préalablement intéressé par cette pratique.

En France, seules deux études empiriques traitent des représentations de la gratuité qu'ont les publics des musées et des monuments et de leurs effets. Ducros et Passebois (2003a et 2003b) abordent la question des représentations de la gratuité par les publics d'une galerie d'art contemporain. Ces chercheurs montrent que la gratuité peut être interprétée symboliquement comme un signal par les consommateurs et ainsi être à même de les insérer dans une relation de don/contre-don avec un prestataire de service ${ }^{6}$. Cependant, cette recherche, limitée à la gratuité permanente mise en œuvre dans une seule galerie d'art contemporain, ne dit rien des effets sur les représentations de l'offre et sur les comportements des publics. Pour leur part, Gottesdiener et Godrèche (1996) ont mis en évidence des effets de la gratuité sur le projet de visite au Louvre : incitation, transfert (report vers les dimanches gratuits) ou contre-transfert (évitement des dimanches gratuits), effets différant en fonction des caractéristiques sociodémographiques des visiteurs. Ces effets ne sont cependant pas expliqués. Ces résultats se limitent de plus aux seuls visiteurs de l'institution atypique qu'est le Louvre. Le peu de travaux dans le champ des musées et des monuments nous a conduit à examiner une littérature plus large sur le comportement du consommateur.

\section{La gratuité et le comportement du consommateur}

\footnotetext{
${ }^{6}$ Toujours dans le champ culturel, celui des échanges de fichiers musicaux sur Napster, Giesler et Pohlmann (2003) arrivent à la même conclusion : la gratuité n'est pas toujours vue comme une absence de prix. Elle peut également être considérée comme une possibilité de don/contre-don et de création de lien.
} 
Là encore, la gratuité d'une offre est très peu considérée. Gorn, Tse et Weinberg (1990) écrivent d'ailleurs : «malgré l'importance de la littérature sur la relation prix-qualité, nous n'avons trouvé aucune étude qui examinerait les perceptions de la qualité du produit ou service lorsqu'il est offert gratuitement ». Ces auteurs conceptualisent la gratuité comme un prix exagérément bas et concluent à un impact négatif sur la qualité perçue, quel que soit le secteur d'activité, marchand ou public. Ce constat, s’il était généralisé au cas des musées et des monuments, irait à l'encontre des présupposés des acteurs culturels, persuadés de l'effet positif de la gratuité, en tant que mesure symbolique, sur le comportement des publics.

Malgré des recherches très nombreuses en promotion des ventes (Chandon, 1994), les outils reposant sur la gratuité ${ }^{7}$ sont peu explorés par comparaison avec les réductions de prix ou les coupons (Chandon, Wansink et Laurent, 2000). Les recherches les plus récentes se focalisent sur les effets de ces outils sur la perception de l'offre, effets différant de ceux des outils promotionnels monétaires : effet positif du «produit en plus » sur la valeur perçue de l'offre (Diamond, 1992 ; Smith et Sinha, 2000 ; Darke et Chung, 2005) et risque de dévalorisation du produit offert lui-même (Raghubir, 2004). D'autres travaux mettent en évidence un effet positif sur la notoriété de la marque en attirant l'attention sur celle-ci (Palazon-Vidal et Delgado-Ballester, 2005) et sur les préférences (Liao, 2006). Ici encore, les effets de la gratuité semblent complexes et parfois contradictoires. Un approfondissement de nos questions de recherche peut donc être utile afin de manier avec justesse cette mesure tarifaire. En définitive, ces études laissent penser, sans permettre de conclure, que les représentations de la gratuité et ses effets sur les comportements sont contrastés. Cette rareté des travaux a

\footnotetext{
${ }^{7}$ La gratuité ne se confond pas avec la promotion. Cette dernière peut être définie comme une modification temporaire et tangible de l'offre dont l'objectif est d'avoir un impact direct sur le comportement des clients de l'entreprise et sur la force de vente (Chandon, 1994). L'aspect promotionnel ne concerne donc que la gratuité périodique et non permanente d'une offre. De plus, seules quelques formes de promotions reposent sur la gratuité : l'offre de produit similaire gratuit considéré comme une promotion monétaire car réduisant le prix de l'offre globale ainsi que la prime (produit ou objet différant de l'offre de base), les techniques d'essai et les échantillons, formes de promotion de type non monétaire.
} 
déterminé le choix de la méthodologie : un objectif exploratoire servi par une stratégie de multiangulation de la production et de l'analyse des données suivant une logique inductive et itérative décrite par Glaser et Strauss (1967) comme la «grounded theory » (théorie enracinée dans les faits) et appliquée notamment par Bergadaà (2006).

\section{METHODOLOGIE DE LA RECHERCHE}

La problématique de la recherche a été formulée de la manière suivante : dans le contexte français, quelles représentations de la gratuité les publics des musées et des monuments, visiteurs et non-visiteurs, ont-ils et comment celles-ci sont-elles reliées à leurs représentations, projets d'usage et comportements de visite de ces lieux ? La contribution attendue étant de générer à partir des données empiriques une connaissance théorique sur ces représentations et leurs effets, la recherche a utilisé une logique inductive (théorie élaborée progressivement au cours de la production et de l'analyse des données sans corpus théorique défini a priori) et itérative (comparaison constante entre les données empiriques et les données théoriques progressivement mobilisées). Afin de servir la qualité des résultats de cette recherche et pour servir l'objectif exploratoire, une stratégie de multiangulation (Denzin, 1978 ; Lewis et Grimes, 1999 ; Gioia et Pitre, 1990 ; Gombault et Hlady-Rispal, 2004) a été adoptée. Toutes les tactiques de multiangulation (des données, des modes de production des données, des théories, des chercheurs et des paradigmes, sauf celle des retours d'analyses ${ }^{8}$ aux personnes étudiées) ont été mises en œuvre.

Insérer Figure 1 - Le schéma de la recherche

Les données ont été collectées dans différentes régions (parisienne et autres, zones urbaines et rurales), les observations réalisées dans des institutions de natures différentes (un musée et un monument) et dans des configurations tarifaires différentes en 2002 et 2003 . La recherche a été effectuée en suivant un schéma précis : un mode central de production et d'analyse des

\footnotetext{
${ }^{8}$ Cette tactique consiste à restituer les analyses aux personnes étudiées pour enregistrer leurs réactions et les analyser à nouveau. Elle aurait été longue et coûteuse à mettre en œuvre.
} 
données - 52 entretiens individuels en profondeur, structurés mais non dirigés - complétés par 4 entretiens de groupe, 36 observations sur site et 580 questionnaires, données collectées auprès de visiteurs ${ }^{9}$ habitués et occasionnels et de non-visiteurs ${ }^{10}$. Le guide d'entretien, identique pour tous les entretiens (individuels, de groupe, de visite) était constitué de deux consignes : «Que représente pour vous la gratuité des musées et des monuments ?» et «Quelles pratiques avez-vous des musées et des monuments?». Chaque consigne était assortie d'un guide thématique permettant d'effectuer les relances. Il se concluait par le scénario de l'offre d'un billet gratuit : «Si l'on vous proposait un billet gratuit pour une visite au musée près de chez vous, dans le mois qui vient, l'utiliseriez-vous ? ».

Une analyse de contenu thématique ${ }^{11}$ de l'ensemble des entretiens retranscrits a été réalisée (Miles et Huberman, 1994, Wacheux 1996). Cette analyse purement qualitative a consisté à transcrire puis à coder manuellement les discours, c'est-à-dire à les réduire en les découpant en fragments porteurs d'une signification, représentant un thème empirique identifié. Un dictionnaire des thèmes empiriques a ainsi été construit. Les entretiens individuels ont fait émerger les principaux thèmes empiriques relatifs aux représentations de la gratuité et à ses effets sur le comportement de visite. Les entretiens de groupe ont légèrement accru la saturation en intégrant les aspects sociaux et la dynamique de groupe sans apporter de réelles contradictions. En contextualisant la production des données, les observations et les entretiens de visite sur site ont apporté une compréhension du vécu de la gratuité. Enfin, conformément à l'objectif exploratoire de la recherche, l'enquête quantitative a permis d'approfondir certains thèmes empiriques.

\footnotetext{
${ }^{9}$ Les caractéristiques des échantillons interrogés sont présentés dans l'annexe 1.

${ }^{10}$ Les visiteurs habitués ont réalisé plus de deux visites dans l'année écoulée, les occasionnels au moins une visite dans les cinq dernières années et les non-visiteurs n'ont pas effectué de visite depuis cinq ans. Seules les personnes vivant en France ont été interrogées.

${ }^{11}$ Une présentation de la méthode d'analyse des données est proposée en annexe 2.
} 
Ensuite, une analyse par inférence théorique (induction et itération) du dictionnaire des thèmes empiriques a conduit à bâtir un dictionnaire des thèmes théoriques, qui recense les concepts et théories susceptibles de contribuer à l'interprétation des thèmes empiriques et ainsi de favoriser leur compréhension. Les théories existantes à propos de la gratuité, du prix, du comportement du consommateur en général et dans le contexte culturel en particulier, ainsi que de théories issues de différentes disciplines - psychosociologie, sociologie, psychanalyse ayant trait aux représentations, à l'argent, à la culture, aux musées et aux monuments ont été mobilisées. Ce dictionnaire final des thèmes théoriques a permis la formulation de 27 propositions théoriques, à valeur d'hypothèses. Celles-ci ont été ensuite reprises, discutées et réduites à trois méta-propositions, généralisables théoriquement (Gergen, 1994).

Le processus de recherche s'est efforcé de satisfaire aux critères de validation propres aux méthodes qualitatives et mixtes (Mucchielli, 1996) : complétude et saturation atteints grâce à la stratégie de multiangulation d'une part, acceptation interne, cohérence interne, confirmation externe atteints grâce aux échanges entre chercheurs, avec le commanditaire et avec la communauté académique d'autre part. Le processus de recherche n'en présente pas moins des limites, notamment sa dimension synchronique (résultats obtenus sur une même période).

\section{PRESENTATION DES RESULTATS}

La recherche menée aboutit à trois métapropositions à valeur d'hypothèses. La première porte principalement sur les représentations de la gratuité et sur leurs effets sur les représentations des musées et des monuments (MP1), la seconde permet de mieux comprendre l'effet de la gratuité sur le projet d'usage de ces lieux (MP2) et enfin la troisième met en évidence les modifications de comportement liées à l'expérience de visite gratuite (MP3). Ce sont ces trois métapropositions que nous présentons et discutons comme résultats de la recherche. 
Conformément à la logique inductive, nous exposons les résultats empiriques ${ }^{12}$ de la recherche et interprétons ceux-ci à la lumière de différentes théories.

MP1 : Les représentations des musées et des monuments s'inscrivent globalement dans un cadre de référence unique, de biens communs culturels dans un système marchand, que la gratuité interroge.

Bien que les musées et les monuments soient décrits par leurs publics comme des biens communs culturels qui, selon une logique collective, doivent être accessibles à tous, ils sont reconnus comme appartenant à un système marchand. Avant tout, les publics se représentent la gratuité dans une logique individuelle, comme une absence de prix, et non dans une logique collective comme une politique culturelle ${ }^{13}$. Ils rapprochent en effet systématiquement la gratuité du fait de payer ou de ne pas payer l'entrée. Plus précisément, l'analyse des discours a permis d'identifier que le paiement de l'entrée est considéré comme un visa d'entrée dans les musées et les monuments, qu'il matérialise une distance culturelle, économique et physique entre les musées et les monuments et leurs publics et qu'il concrétise leur engagement dans l'acte de visite. Par conséquent, les publics considèrent que la gratuité supprime ce visa, réduit cette distance et rend l'engagement dans la visite moins formel. De plus, la gratuité est discutée sous un angle très matérialiste en étant associée à une opportunité, voire une «bonne affaire », ou à un gâchis. Enfin, les publics rapprochent la gratuité de la valeur accordée aux musées, aux monuments et à leur visite : élément de valorisation pour les uns, de dévalorisation pour les autres, ou élément sans lien avec leur valeur.

\footnotetext{
${ }^{12}$ Les passages en italique sont des extraits d'entretiens.

${ }^{13}$ Ce cadre de référence apparaît ainsi très éloigné de la conception des acteurs institutionnels qui voient généralement la gratuité comme une valeur fondatrice de l'idéologie originelle des musées et des monuments (Gombault, 2002) ou encore des représentations de la gratuité comme un don mises en évidence par Ducros et Passebois (2003a et 2003b).
} 
Sous cet angle, deux paradoxes intra-individuels apparaissent. D'une part, pour plusieurs personnes, plutôt favorables à la gratuité, le fait de ne pas payer l'entrée, d'une façon générale, est important. Ils considèrent que la gratuité va favoriser la fréquentation, pour les autres, mais beaucoup moins pour eux. Ils évoquent alors le manque de temps, les contraintes individuelles qui les empêchent de profiter de la gratuité. Ce premier paradoxe montre une vision contradictoire de la gratuité selon que l'on se la représente pour soi ou pour les autres. D'autre part, certaines personnes, se déclarant souvent défavorables à la gratuité, considèrent qu'elle dévalorise les musées et les monuments et leur visite, notamment en dégradant les conditions de visite (dépréciation des sites, foule, présence de badauds...). Elles l'associent à un gâchis tout en affirmant qu'elle ne développera pas la fréquentation. Ces mêmes personnes s'accordent cependant sur le statut de biens communs culturels devant être accessibles à tous. Des divergences inter-individuelles fortes sont également constatées. $62,6 \%$ des individus sont d'accord avec la proposition selon laquelle «L'entrée des musées et des monuments doit, par principe, être gratuite. ». C'est le mode de tarification plébiscité par 21,5\% d'entre eux. 86,3\% des répondants sont d'accord avec le principe d'une participation symbolique, $67 \%$ plébiscitant ce mode de tarification. Enfin, 28,1\% des individus sont d'accord avec la proposition selon laquelle «L'entrée des musées et des monuments doit, par principe, être payante. » et le prix est le mode de tarification proposé en premier lieu par $6 \%$ des individus. Ainsi, pour certains, l'accessibilité suppose la gratuité de l'entrée. Pour d'autres, le paiement d'un prix symbolique concrétise l'attachement à un patrimoine collectif, la participation à son entretien et l'engagement dans la visite. Pour d'autres encore, ce patrimoine se mérite, ce qui justifie le paiement de l'entrée, ou, dernier cas de figure, la visite des musées et des monuments est un loisir comme un autre qui se paie, ou qui, par opportunité peut être gratuit. Dès lors, le débat s'ouvre sur la question du bien-fondé de la gratuité : faut-il ou non rendre 
les musées et les monuments accessibles en les rendant gratuits? Logiques collective et individuelle se confrontent.

Ces représentations contrastées d'un bien culturel collectif dont l'usage (la visite) fait l'objet d'un prix, résultent d'un conflit entre des représentations de l'argent (Urbain, 2000, 2002), fortement imprégnées de morale (Sédillot, 1989), des valeurs collectives et individuelles en évolution et des représentations très idéologiques de la culture. En supprimant le visa d'entrée que représente le fait de payer, la gratuité réduit la distance entre les musées et les monuments et le visiteur qu'introduisaient, dans un processus d'objectivation, le paiement et l'argent (Reiss-Schimmel, 1992 ; Simmel, 1907). Les effets contradictoires concernant la valeur de l'objet gratuit mis en évidence dans les recherches sur les promotions non monétaires (Diamond, 1992 ; Smith et Sinha, 2000 ; Darke et Chung, 2005 ; Raghubir, 2004) sont confirmés ici.

Ces représentations de la gratuité sont liées à la diversité et la dynamique des représentations qu'ont les publics des musées et des monuments. Ces derniers sont pensés globalement dans un cadre de référence unique: des biens communs culturels proposés dans un système marchand. Quatre univers fragmentent ce cadre de référence: un univers sacré, représentation originelle des musées et des monuments, marqué par une vision dogmatique et esthétique et dans lequel la gratuité est considérée comme naturelle ; un univers patrimonial au sens culturel mais également au sens économique et juridique, dominant aujourd'hui, dans lequel la gratuité, très controversée, apparaît comme un idéal impossible; un univers de loisirs qui offre des prestations de services dans une logique de consommation dans laquelle la gratuité est considérée comme une opportunité, une promotion ; un dernier univers, dans lequel les musées et les monuments sont absents, où la gratuité tout comme le prix n'ont pas de sens. L'identification de l'univers loisirs chez les publics français rejoint les travaux 
américains sur «l'entertainment economy» ou «experience economy» (Falk, 1994 ; Pine et Gilmore, 1999 ; Wolf, 1999).

MP2 : La gratuité est secondaire dans la construction et la réalisation d'un projet de visite.

Les données empiriques montrent que ces représentations contrastées de la gratuité ont des effets sur les intentions de visite des publics, la gratuité ne suffisant cependant pas à elle seule à créer l'envie de visiter ces lieux. Ainsi les visiteurs habitués disent que «la gratuité ne fera venir personne ou si peu de personnes », que «c'est un faux problème ».

La recherche montre que les représentations de la gratuité s'intègrent dans leurs représentations du prix de la visite. Celui-ci est perçu comme un prix global : sont non seulement mentionnés le prix payé à l'entrée mais également les dépenses de déplacement (transports, parking...), voire d'hébergement i.e. les efforts monétaires générés par la visite. Les personnes abordent aussi les désagréments liés à la visite tels que la nécessité de décider puis d'organiser la visite, le risque de déception et l'effort intellectuel demandé, la crainte de la foule ou d'une ambiance peu agréable, la fatigue physique anticipée ; en d'autres termes, les efforts non monétaires de la visite - psychologiques et physiques. Les publics expriment ainsi une acception élargie du prix, considéré comme ce qui est abandonné ou sacrifié pour obtenir un produit (Zeithaml, 1988). Le prix de la visite comprend une composante monétaire et une composante non monétaire (Murphy et Enis, 1986).

La composante non monétaire du prix, malgré la gratuité de l'entrée, est particulièrement relevée : l'effort intellectuel, le temps dont il faut disposer, l'organisation de la visite. D’autres efforts non monétaires sont renforcés, voire créés, par la gratuité : la foule, la présence de personnes non intéressées ou différentes du public habituel, la nécessité de prévoir la visite pour profiter de la gratuité. Dans ce contexte, la gratuité du prix d'entrée représente uniquement la suppression de l'un des efforts monétaires directs de la visite : une absence de prix d'entrée au sein d'un prix global. Les publics soulignent alors que la gratuité 
n'existe pas réellement : puisque la visite des musées et des monuments, même sans prix d'entrée, entraîne toujours un effort quelconque, monétaire ou non, elle n'est jamais gratuite. L'importance est ainsi rappelée, dans le contexte des musées et des monuments, des éléments non monétaires du prix, déjà soulignés par Bailey et Falconer (1998), Fines (1981) et Mc Lean (1997). Les conclusions de Prottas (1981) sont confirmées : les composantes non monétaires du prix ont une importance d'autant plus grande qu'un service est gratuit, là où pourtant on penserait spontanément qu'il n'existe plus de barrières à l'entrée. En supprimant le prix, la gratuité met en lumière les autres coûts qui pèsent sur la décision de visite et qui sont autant de freins à sa réalisation. Ces éléments corroborent les résultats relatifs à l'impact négatif de la gratuité sur la qualité perçue mis en évidence dans le domaine promotionnel (Gorn, Tse et Weinberg, 1990).

Ces considérations sur la véritable nature de la gratuité amènent les individus à exposer l'effet que cette mesure peut avoir sur leurs intentions de visite. Une contribution importante de la recherche est de mettre en évidence que la gratuité ne crée pas d'intention de visite. Elle facilite simplement le passage à l'action lorsque l'intention de visite existe préalablement.

En premier lieu, la gratuité permet tout de même de faire penser aux musées et aux monuments comme une activité dominicale potentielle. En second lieu, la gratuité améliore l'accessibilité objective (financière et physique) et symbolique de l'offre des musées et des monuments. Lorsque l'entrée est gratuite, les musées et monuments ne sont plus perçus comme à l'ordinaire. On s'éloigne de la vision normée de la visite, avec ses règles comportementales, son intention et ses obligations de rentabilité culturelle. En troisième lieu, la gratuité change le processus de décision de visite. Lorsque le musée ou le monument est gratuit, le processus de prise de décision est plus simple, spontané.

Nous retrouvons ici les effets positifs de la gratuité sur la notoriété de l'objet concerné mis en évidence par Palazon-Vidal et Delgado-Ballester, (2005). Il est aussi possible de dire que le 
processus de décision est modifié. Dans un cadre de gratuité, le consommateur déclare, en effet, ne plus avoir besoin a priori d'évaluer la pertinence d'une consommation en opérant une recherche d'information importante. La gratuité permet de «consommer» le lieu culturel différemment et interviendrait comme un stimulant de la tendance exploratoire du consommateur. Ceci suggère que le consommateur adopte, grâce à la gratuité, un comportement expérientiel de type exploratoire (Berlyne, 1960; Holbrook et Hirschman, 1982 ; Bourgeon, 2005).

MP3 : L'expérience de la visite gratuite des musées et des monuments peut permettre un apprentissage de leur visite ainsi que l'appropriation de la mesure de gratuité.

Enfin pour réduire la dissonance créée par l'existence de mesures de gratuité et l'expérience de la gratuité, les personnes réagissent de deux façons. La majeure partie d'entre elles restent dans le cadre de référence de l'échange et rejettent la gratuité : elles renforcent leurs représentations préexistantes à la gratuité. Les autres, ayant fait l'expérience de la gratuité, font évoluer ce cadre de référence.

Ainsi, les données empiriques montrent combien le fait de vivre une visite gratuite représente une expérience déconcertante pour certains visiteurs habitués au paiement (pas pour tous cependant). La gratuité les conduit à vivre une expérience où leur comportement est plus détendu, voire plus profane. Mais au-delà de cette déconstruction des repères habituels, la visite gratuite peut correspondre à une visite différente de celle qui était attendue. Loin de correspondre aux représentations négatives généralement associées à cette forme tarifaire, la visite gratuite est une nouvelle expérience dans le sens où elle favorise un sentiment de liberté, stimule le lien social et modifie l'appropriation des lieux et les ressentis. Les représentations des musées et des monuments, les buts de leur visite et les modalités de leur découverte en sont modifiés. Ils sont alors perçus comme plus accessibles. Visiter un lieu 
culturel quand il est gratuit donne lieu à un projet d'usage moins rigide et moins synonyme d'efforts cognitifs, c'est-à-dire moins «culturel » comme le disent les personnes rencontrées. Ainsi la mesure de gratuité est apparue comme un élément de déconstruction des représentations et comportements liés à la pratique habituelle de visite des musées et des monuments. Il se met en place un processus d'apprentissage de la gratuité, qui peut, par effet boomerang, conduire à un apprentissage de la pratique de visite. Le visiteur réduit la dissonance cognitive liée aux représentations initiales négatives selon un processus qui peut être qualifié d'apprentissage par l'expérimentation (Kolb, 1984).

A l'inverse, pour ceux qui n'ont pas expérimenté la gratuité, les représentations de la visite gratuite et des publics en bénéficiant, restent le plus souvent négatives (c'est également le cas de quelques visiteurs expérimentant la gratuité, mais restant très attachés au paiement). Pour réduire les dissonances cognitives, ceux qui n'ont pas vécu la gratuité s'attachent à l'éviter pour conforter leurs certitudes et sont alors les plus critiques vis à vis de cette mesure. Il y a donc un phénomène d'évitement de la pratique, une logique de renforcement des représentations négatives de la mesure, et une argumentation basée sur la discrimination des publics («ce n'est pas pour moi mais pour les autres ») pour ceux qui n'ont pas expérimenté la gratuité.

Les résultats montrent que l'expérience de la gratuité peut permettre l'apprentissage de la pratique de visite. En permettant à des non-visiteurs de découvrir ce qu'ils peuvent vivre au cours de la visite d'un musée ou de monument, l'expérience de la visite gratuite pourrait provoquer chez eux le «déclic culturel» attendu par les professionnels. Ces visiteurs pourraient vivre une première fois ce type d'expérience, en d'autres termes pourraient « essayer » la visite d'un musée et/ou d'un monument. L'expérience effective d'une visite culturelle gratuite conduirait ensuite à rechercher d'autres expériences de visite se fondant sur l'approche expérientielle (Bourgeon et alii, 2006). En effet, lors de la visite, l'individu vit des 
éléments nouveaux d'une expérience qui imposent des ajustements sur ses représentations et sa perception préalables de l'activité. Il réduit ainsi ses dissonances cognitives (Festinger, 1957), processus à relier à la théorie du conditionnement instrumental (Skinner, 1950). Si le comportement de consommation conduit à un résultat positif (une récompense), la probabilité qu'il soit adopté augmente (renforcement positif). L'apprentissage se réalise alors selon le principe d'addiction, important dans les habitudes de consommation culturelle : «plus l'individu consomme une activité culturelle avec plaisir, plus il a le désir et le goût de consommer cette activité ».

IMPLICATIONS, LIMITES ET VOIES DE RECHERCHE

Les résultats présentés ici visaient à répondre à la problématique suivante : dans le contexte français, quelles représentations de la gratuité les publics des musées et des monuments ontils et comment celles-ci sont-elles reliées à leurs représentations, projets d'usage et comportements de visite? Les trois métapropositions à valeur d'hypothèses, aboutissement de la recherche, contribuent à la compréhension des représentations qu'ont les publics de la gratuité et de leurs effets :

- Notre premier résultat précise que les représentations des musées et des monuments s'inscrivent globalement dans un cadre de référence unique, de biens communs culturels dans un système marchand, que la gratuité déstabilise. Ce résultat montre que les représentations de la gratuité sont aussi fonction de l'objet auquel elle est appliquée. Ainsi, les contrastes mis en évidence résultent de représentations des musées et monuments comme des lieux sacrés, comme un patrimoine commun ou pour certains comme un loisir. Ce résultat est fortement lié à la dimension culturelle et publique de notre objet de recherche. Ces résultats ont des implications managériales fortes. Ils montrent notamment la nécessité d'expliciter la finalité de chaque institution, de les situer dans un univers de représentation : expositions «pointues » pour public initié, mise à disposition d'un patrimoine commun dans un lieu ouvert à tous, 
positionnement comme un loisir alternatif.... Ils mettent également en évidence la nécessité de différencier les politiques tarifaires (gratuité, tarif ou prix) en fonction de ces finalités.

- Notre second résultat montre que la gratuité est secondaire dans la construction et la réalisation d'un projet de visite. La gratuité ne suffit pas à créer le désir de consommer ; elle permet uniquement de mettre au jour des intentions latentes. Ce résultat semble potentiellement généralisable à tout produit ou service. Même gratuit, n’importe quel produit n'en est pas pour autant désirable si le consommateur n'est pas impliqué dans sa consommation. Ici encore ce résultat est d'importance sur un plan managérial. Il souligne la nécessité d'accroître l'implication des publics vis à vis de cette activité et le besoin de communiquer davantage sur les mesures de gratuité afin de révéler les intentions de visite latentes. Il faudrait également mettre en œuvre une gestion des coûts non monétaires liés à la visite, mis en exergue voire créés par la situation de gratuité afin d'éliminer des freins à la visite. Une politique tarifaire de gratuité est donc susceptible d'entraîner des coûts (au-delà du fait qu'elle supprime des revenus) si l'institution ne souhaite pas que la qualité perçue de la visite en souffre.

- Enfin notre troisième et dernier résultat met en évidence que l'expérience de la visite gratuite des musées et des monuments peut permettre un apprentissage de leur visite ainsi que l'appropriation de la mesure de gratuité par certains visiteurs. La gratuité permet l'essai et peut provoquer l'attachement à l'objet gratuit, mais aussi à la gratuité. Ce résultat semble également généralisable à tout type de bien ou de service, des effets de fidélité à la promotion ayant d'ailleurs été mis en évidence dans le secteur marchand (Desmet, 2002). La mise en évidence d'un apprentissage souligne la nécessité de réfléchir à la continuité de la mesure dans le temps, de penser à mettre en place des rituels de fréquentation autour de la gratuité. Il semble également nécessaire de mettre en avant sa dimension festive, exceptionnelle (comme cela peut être fait dans le cas des Journées du patrimoine ou de la Nuit des musées), de 
préciser quels en sont les segments visés, ceci afin de réduire les présupposés négatifs à son encontre.

Ces résultats interpellent ainsi les acteurs institutionnels principalement sur deux points :

- D'une part sur le besoin d'accroître l'implication dans l'activité de visite. Il faut le répéter : la gratuité facilite ou permet la visite, mais elle est loin d'être la clé d'entrée des musées et des monuments. Les pistes à suivre pour ces acteurs seraient avant tout de repérer les leviers permettant d'accroître l'intérêt pour la pratique en initiant à la visite, l'attractivité des lieux ainsi que la pertinence de leur visite en tant que loisir alternatif. Ceci doit être fait en prenant en compte la diversité des publics et de leurs représentations de ces lieux. Il s'agirait également de construire une image de lieux plus animés et plus vivants, permettant aux visiteurs de vivre des émotions, de partager une expérience...(Bourgeon et alii, 2006).

- D'autre part sur la nécessité de mieux accompagner la mesure de gratuité, à la fois d'en faire une véritable politique ou stratégie et quand celles-ci existent d'en donner clairement le sens : Pourquoi appliquer la gratuité ? De quels choix relève-t-elle ? Quelle est la stratégie suivie ? Quels sont les buts à atteindre ? Comment est-elle mise en œuvre ? Les bénéficiaires insistent sur la nécessité d'inscrire cette démarche dans le temps et de la généraliser à l'ensemble des musées et monuments de France pour qu'elle soit complètement intégrée dans les habitudes de la société actuelle, compte tenu du besoin d'apprentissage progressif tant de la mesure de gratuité que de l'activité culturelle. L'autre choix stratégique serait d'en faire un événement périodique ou ponctuel très médiatisé, en prenant comme exemple les Journées du Patrimoine. Sa mise en œuvre nécessite également de prendre des mesures de réduction des désagréments cités par les publics (foule, diversité des publics, nécessité de prévoir et de s'organiser...).

Cette recherche comporte également des limites et ouvre des voies de recherche. 
D’une manière générale, elle offre un état des lieux qui peut servir de point de référence pour des investigations ultérieures. Une étude diachronique permettrait de vérifier le degré de stabilité des observations dans le temps, et notamment de contrôler l'effet à long terme de la mesure sur les pratiques (essai et fidélité). Il serait également intéressant d'obtenir des résultats selon les types d'offre (notoriété des sites, positionnement, etc.). Par ailleurs, le repérage d'univers qui structurent les représentations des musées, des monuments et de leur gratuité est un résultat qui mériterait d'être lui aussi approfondi en regardant le processus de structuration de ces représentations et en identifiant les variables intervenantes par une analyse typologique des publics.

Plus généralement, cette recherche interroge les pratiques de prix de toute organisation marchande ou non. Elle pourrait être poursuivie en étudiant les différents types de gratuité (permanente ou périodique, catégorielle ou non, totale ou partielle) et les objectifs qui peuvent leur être assignés (faire essayer, acheter, racheter, faire venir sur le point de vente, former et informer...), en considérant leur adéquation à différentes cibles et à différents types de produit (impliquant ou non, familier ou non...). Par ailleurs, il serait important de mesurer quels sont les coûts non monétaires mis en exergue dans un contexte de gratuité afin de mieux évaluer l'impact de la gratuité sur la valeur perçue globale (Zeithaml, 1988 ; Aurier, Evrard et N'Goala, 2004). Il serait également intéressant d'explorer l'influence de la gratuité sur la perception future des prix et notamment sur la formation des prix de référence (Zollinger, 2004) ou le consentement à payer (Le Gall, 2000). Enfin, il faut rappeler la spécificité de notre terrain d'application, les musées et les monuments français (service public avec paiement indirect, avec effets d'externalités négatifs en cas d'affluence, produit socialement connoté). Les questions que pose la gratuité, mériteraient d'être étudiées dans d'autres champs culturels (musique, photographies, journaux) et d'autres services publics (transport, bibliothèque, éducation, santé...) ou privés utilisant la gratuité comme outil promotionnel. 


\section{BibLIOGRAPHIE}

Abric J.C. (1993), Représentations sociales et pratiques, Paris, PUF.

Aurier P., Evrard Y. et N'Goala G. (2004), Comprendre et mesurer la valeur du point de vue du consommateur, Recherche et Applications en Marketing, 19, 3, 1-20.

Bagdali S. (1998), Il museo come azienda. Management e organizzazione al servizio della cultura, Milano, ETAS.

Bailey S., Falconer P., Foley M., McPherson G. et Graham M. (1997), Charging for admission to museums and galleries : arguments and evidence, Museum Management and Curatorship, 16, 4, 355-369.

Bailey S., et Falconer P. (1998), Charging for admission to museums and galleries : a framework for analysing the impact on access, Journal of Cultural Economics, 22, 2, 167 177.

Bergadaà M. (2006), Le don d'objets : dimensions centrales et profils de donneurs aux œuvres de bienfaisance, Recherche et Applications en Marketing, 21, 1, 19-39.

Berlyne D.E. (1960), Conflict, arousal and curiosity, Series in Psychology, New-York, McGraw-Hill Book Company.

Bourgeon D. (2005), Du marketing expérientiel appliqué aux musées, Revue Espaces, 87, 4147.

Bourgeon D., Petr C., Gombault A., Le Gall-Ely M. et Urbain C. (2006), An experiential approach to the consumption value of arts and culture : the case of museums and monuments, International Journal of Arts Management, 9, 1, 35-47.

Chandon P. (1994), Dix ans de recherches sur la psychologie et le comportement des consommateurs face aux promotions, Recherche et Applications en Marketing, 9, 2, 83-108.

Chandon P., Wansink B. et Laurent G. (2000), A benefit congruency framework of sales promotion effectiveness, Journal of Marketing, 64, 4, 65-81.

Darke P.R. et Chung C.M.Y. (2005), Effects of pricing and promotion on consumer perceptions : it depends on how you frame it, Journal of Retailing, 81, 1, 35-47.

Denzin N. (1978), Interpretative biography, Newbury Park, CA, Sage.

Desmet P. (2002), Promotion des ventes, 2 ème édition, Paris, Dunod.

Desmet P. et Zollinger M. (1997), Le prix: de l'analyse conceptuelle aux méthodes de fixation, Paris, Dunod.

Diamond W.D. (1992), Just what is a «dollar's worth»? Consumer reactions to price discounts vs. extra product promotions, Journal of Retailing, 68, 3, 254-270.

Dickenson V. (1993), The economics of museum admission charges, Curator, 36, 3, 220-234. Ducros L. et Passebois J. (2003a), Les effets de la gratuité sur la fréquentation des équipements culturels: une réflexion à travers la théorie du «don contre don», 7th International Conference on Arts management (AIMAC), Milan, Italy, Bocconi University.

Ducros L. et Passebois J. (2003b), Pour un marketing de l'altruisme : une application aux

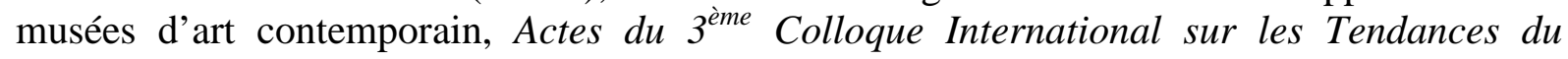
Marketing, Universita Ca' Foscari, Venezia.

Falk J.H. (1994), The museum experience, Washington DC, Whalesback Books.

Festinger L. (1957), A theory of cognitive dissonance, Palo Alto, CA, Stanford University Press.

Fines S.H. (1981), The marketing of ideas and social issues, New-York, Praier.

Fourteau C. (2001), La gratuité au bois dormant... Cinq ans de gratuité du dimanche au Louvre, 1996-2000., Paris, Editions du service culturel du musée du Louvre.

Gergen K. (1994), Realities and relationships. Soundings in social construction, Cambridge, Mass., Harvard University Press. 
Giesler M. et Pohlmann M. (2003), The anthropology of file sharing : consuming Napster as a gift, Advances in Consumer Research, 30, 1, Provo, Utah, Association for Consumer Research, 273-279.

Glaser B. et Strauss A. (1967), The discovery of grounded theory, Chicago, IL, Aldine.

Godbout J.T. (2000), Le don, la dette, l'identité, Paris, La découverte.

Godbout J.T. et Caillé A. (1992/2000), L'esprit du don, Paris, La découverte.

Goia D.A. et Pitre E. (1990), Multiparadigm perspectives on theory building, Academy of Management Review, 15, 4, 584-602.

Gombault A. (2002), La gratuité dans les musées : une revue internationale, Les institutions culturelles au plus près du public, éd. Fourteau C., Paris, La Documentation Française, 193228.

Gombault A. et Hlady Rispal M. (2004), Multiangulation in qualitative research : necessity and risks, International Conference on Research Methods "Crossing frontiers in quantitative and qualitative research methods", Research Methods Division, Academy of Management / ISEOR, Université Jean Moulin Lyon 3.

Gombault A., Petr C., Bourgeon D., Le Gall-Ely M. et Urbain C. (2006), La gratuité des musées et monuments côté publics, Paris, La documentation française.

Gorn G.J., Tse D.K. et Weinberg C.B. (1990), The impact of free and exaggerated prices on perceived quality of services, Marketing Letters, 2, 2, 99-110.

Gottesdiener H. et Godrèche N. (1996), Les dimanches gratuits du musée du Louvre, enquête qualitative, Paris, Musée du Louvre-service culturel, CEREM.

Hénaff M. (2002), Le prix de la vérité. Le don, l'argent, la philosophie, Paris, Seuil.

Hirschman E.C. et Holbrook M.B. (1982), Hedonic consumption : emerging concepts, methods and propositions, Journal of Marketing, 46, 3, 92-101.

Jodelet D. (éd.) (1997), Les représentations sociales, Paris, PUF.

Jouvenel (de) B. (2002), L'économie politique de la gratuité (1957), Futuribles, 281, 73-82.

Kolb D.A. (1984), Experiential learning, experience as the source of learning and development, Englewood Cliffs, N.J., Prentice Hall.

Le Gall M. (2000), Contribution à l'évaluation monétaire de biens et services sans référent de marché : les apports combinés de la méthode de l'évaluation contingente et de l'analyse des mesures conjointes, Thèse de Doctorat en Sciences de Gestion, Université de Rennes 1.

Lewis M.W. et Grimes A.J. (1999), Metatriangulation : building theory from multiple paradigms, Academy of Management Review, 24, 4, 672-690.

Liao S.-L. (2006), The effects of nonmonetary sales promotions on consumer preferences : the contingent role of product category, Journal of American Academy of Business, 8, 2, 196203.

Mauss M. (1923-1924), Essai sur le don, Paris, PUF.

McLean F. (1997), Marketing the museum, London, Routledge.

Miles M. et Huberman A. (1994), Qualitative data analysis : an expanded sourcebook, Berverly Hills, CA, Sage Publications.

Monroe K.B. (1990), Pricing : making profitable decisions, New York, Mc GrawHill.

Mucchielli A. (éd.) (1996), Dictionnaire des méthodes qualitatives, Paris, Armand Colin.

Murphy P.E. et Enis B.M. (1986), Classifying products strategically, Journal of Marketing, $50,3,24-42$.

Octobre S. et Rouet. F. (2002), La gratuité du premier dimanche du mois dans les monuments et musées nationaux, Les tarifs de la Culture, Rouet F. (éd.), Paris, La documentation Française, 303-337.

O'Hagan J. (1995), National museums : to charge or not to charge ?, Journal of Cultural Economics, 19, 1, 33-47. 
O'Hare M. (1975), Why do people go to museums? The effect of prices and hours on museum utilization, Museum, 27, 3, 134-146.

Palazon-Vidal M. et Delgado-Ballester E. (2005), Sales promotions effects on consumerbased brand equity, International Journal of Market Research, 47, 2, 179-204.

Pine J.B. et Gilmore J.H. (1999), The experience economy: work is theatre and every business a stage, Boston, Harvard Business School Press.

Prottas J.M. (1981), The cost of free services : organization impediments of access to public services, Public Administration Review, 41, 5, 526-534.

Raghubir P.(2004), Free gift with purchase : promoting or discounting the brand?, Journal of Consumer Psychology, 14, 1-2, 181-186.

Reiss-Schimmel I. (1992), Freud, la psychanalyse et l'argent, Thèse de Doctorat en Sciences Humaines, Université Paris V - René Descartes - Sorbonne.

Sagot-Duvauroux J.L. (1995), Pour la gratuité, Paris, Desclés De Brouwer.

Sédillot R. (1989), Histoire morale et immorale de la monnaie, Paris, Bordas Culture.

Simmel G. (1907), La philosophie de l'argent, traduit de l'allemand, 1987, Paris, PUF.

Skinner B.F.(1950), Are theories of learning necessary?, Psychological Review, 57, 4, 93-216.

Smith M.F.et Sinha I. (2000), The impact of price and extra product promotions on store preference, International Journal of Retail and Distribution Management, 28, 2, 83-92.

Urbain C. (2000), L'attitude à l'égard de l'argent : une première tentative de validation de deux échelles de mesure américaines dans un contexte culturel français, Recherche et Applications en Marketing, 15, 3, 3-28.

Urbain C. (2002), L'attitude à l'égard de l'argent dans le contexte du prix : concepts, mesures et effets, Thèse de Doctorat en Sciences de Gestion, Université de Rennes 1.

Wacheux F.(1996), Méthodes qualitatives et recherche en gestion, Paris, Economica.

Weick K.E. (1989), Theory construction as disciplined imagination, Academy of Management Review, 14, 4, 516-531.

Wolf M.J. (1999), Entertainment economy. How mega-media forces are transforming our lives, New York, Times Books and Random House.

Zeithaml Z. (1988), Consumers perceptions of price quality and value : a means-end model and synthesis of evidence, Journal of Marketing, 52, 3, 2-22.

Zollinger M. (2004), Le jugement comparatif des prix par le consommateur, Recherche et Applications en Marketing, 19, 2, 73-97. 


\section{Annexe 1 - Les caractéristiques des échantillons interrogés}

\begin{tabular}{|c|c|c|c|c|}
\hline $\begin{array}{c}\text { Caractéristiques des } \\
\text { échantillons }\end{array}$ & Entretiens individuels & Entretiens de groupe & $\begin{array}{c}\text { Observations et } \\
\text { entretiens de visite }\end{array}$ & Enquête \\
\hline Taille & 52 entretiens & 4 entretiens & $\begin{array}{c}18 \text { observations au } \\
\text { Musée Magnin (Dijon) } \\
18 \text { au Monument } \\
\text { National de } \\
\text { Chateauneuf en auxois } \\
12 \text { observations lors } \\
\text { des Journées du } \\
\text { Patrimoine (gratuité } \\
\text { exceptionnelle et } \\
\text { médiatisée) } \\
13 \text { un dimanche gratuit } \\
\text { (gratuité périodique) } \\
11 \text { un dimanche payant }\end{array}$ & 580 questionnaires \\
\hline Lieu d'habitation & $\begin{array}{l}12 \% \text { Paris et sa région } \\
54 \% \text { Ville de province } \\
34 \% \text { Zone rurale }\end{array}$ & Dijon & $\begin{array}{l}\text { Habitants et touristes } \\
\text { de la région dijonnaise }\end{array}$ & $\begin{array}{c}17 \% \text { Paris et sa région } \\
48 \% \text { Ville de province } \\
35 \% \text { Zone rurale }\end{array}$ \\
\hline Fréquentation & $\begin{array}{c}40 \% \text { d'habitués } \\
36 \% \text { d'occasionnels } \\
24 \% \text { de non-visiteurs }\end{array}$ & $\begin{array}{c}\text { G1 }: 10 \text { habitués } \\
\text { G2 }: 8 \text { occasionnels } \\
\text { G3 }: 9 \text { habitués } \\
\text { G4 }: 5 \text { non-visiteurs }\end{array}$ & $\begin{array}{c}\text { 66\% d'habitués } \\
31 \% \text { d'occasionnels } \\
3 \% \text { de non-visiteurs }\end{array}$ & $\begin{array}{c}54 \% \text { d'habitués } \\
38 \% \text { d'occasionnels } \\
8 \% \text { de non-visiteurs }\end{array}$ \\
\hline Sexe & $\begin{array}{l}46 \% \text { de femmes } \\
54 \% \text { d'hommes }\end{array}$ & $\begin{array}{l}\text { G1 }: 6 \text { f./4 h. } \\
\text { G2 }: 4 \text { f./4 h. } \\
\text { G3 }: 5 \text { f./4 h. } \\
\text { G4 : } 1 \text { f./4 h. }\end{array}$ & $\begin{array}{l}34 \% \text { de femmes } \\
66 \% \text { d'hommes }\end{array}$ & $\begin{array}{l}60 \% \text { de femmes } \\
40 \% \text { d'hommes }\end{array}$ \\
\hline $\begin{array}{c}\hat{\text { Age }} \\
\text { Min, max, moyenne }\end{array}$ & {$[17 ; 81] 44$ ans } & $\begin{array}{l}\text { E1 : [59; 75], } 66 \text { ans } \\
\text { E2 : [20;46], } 36 \text { ans } \\
\text { E3 : [29; 47], } 35 \text { ans } \\
\text { E4 : [22;31], } 28 \text { ans }\end{array}$ & $\begin{array}{c}\text { Critère remplacé par la } \\
\text { notion d'unité } \\
\text { d'observation : } \\
\text { personne seule (5), } \\
\text { unité familiale (20), } \\
\text { unité amicale (11). }\end{array}$ & $\begin{array}{c}\text { Moins de } 18 \text { ans }=1 \% \\
\text { De } 18 \text { à } 25 \text { ans }=26 \% \\
\text { De } 26 \text { à } 35 \text { ans }=15 \% \\
\text { De } 36 \text { à } 45 \text { ans }=17 \% \\
\text { De } 46 \text { à } 55 \text { ans }=19 \% \\
\text { De } 56 \text { à } 65 \text { ans }=11 \% \\
66 \text { ans et plus }=10 \%\end{array}$ \\
\hline
\end{tabular}




\section{Annexe 2 - L'analyse de contenu thématique des données}

Les extraits proposés ci-dessous illustrent la méthode d'analyse de contenu thématique : du codage des entretiens à la constitution du dictionnaire des thèmes empiriques puis du dictionnaire des thèmes théoriques.

\section{Extrait d'une fiche d'analyse par entretien}

\section{REPRES-/PRIX.MUS\&MON=CHER: $p .1,3,4,5$}

Verbatim p.5

E22: Mais c'est vrai que c'est cher; enfin les loisirs à la base c'est quand même un luxe entre guillemets, même si ça devient plus abordable, on peut pas aller faire n'importe quelle activité si on veut.

\section{Extrait du dictionnaire des thèmes empiriques ${ }^{14}$}

\section{GRATUITE ET ACCESSIBILITE}

- La gratuité et l'accès des musées et aux monuments à différents publics

$[\ldots]$

REPRES/DECIS.GRAT/POUV.PUBL/MOTIFS=OUVERT.A.TOUS:

Les responsables des musées et des monuments décident de proposer la gratuité pour ouvrir les musées et des monuments à tous

E9: p.9; EC16: p.5

REPRES-PAYER/ELITISME:

Le fait de payer les visites des musées et des monuments est élitiste: les personnes qui ont les moyens sont privilégiées

EC11: p.2, 3; E6: p.5; E7: p.1

\section{Extraits du dictionnaire des thèmes théoriques ${ }^{15}$}

I) - COMMENT LA GRATUITE DES MUSEES ET DES MONUMENTS EST-ELLE APPREHENDEE ? UNE APPROCHE GENERALE

A) Les représentations de la gratuité des musées et des monuments dans une logique individuelle

a) La gratuité: un prix

* Le prix de la visite: un prix global

Dico Empirique

Partie I Gratuité, fait de payer et prix

La cherté des prix des musées et des monuments [10 et 11]

Le consentement à payer pour les visites des musées et des monuments [11 et 12]

Partie IV gratuité et budget

La place des dépenses de visites de musées et des monuments dans le budget [25]

$[\ldots]$

P0) Dans les représentations, la gratuité fonctionne comme un prix, c'est à dire comme l'absence de coûts monétaires et non monétaires

P1) Dans les représentations des publics, la gratuité se manifeste comme un prix: elle est envisagée comme une suppression d'un coût monétaire et peut modifier la perception des coûts non monétaires

\footnotetext{
${ }^{14}$ Le dictionnaire des thèmes empiriques est organisé en arborescence, celle-ci permet de remonter des thèmes empiriques au codage des entretiens qui ont permis de les produire.

${ }^{15}$ Le dictionnaire des thèmes théoriques est organisé en arborescence, celle-ci permet de remonter des thèmes théoriques aux thèmes empiriques qui ont permis de les produire.
} 
Figure 1 - Le schéma de la recherche

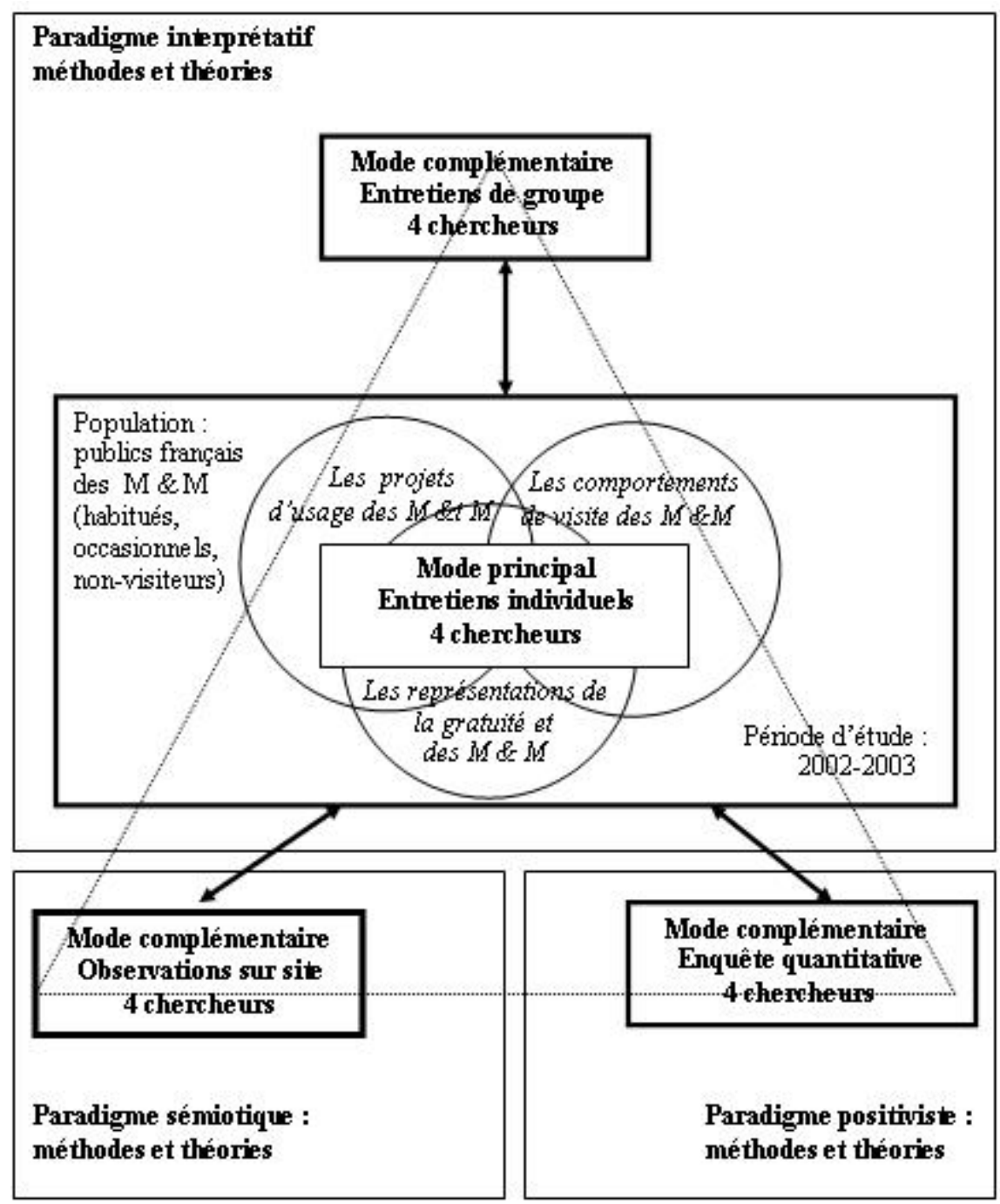

